\title{
Applications of ferroelectrics in photovoltaic devices
}

\author{
Yang Liu ${ }^{1}$, Shufeng Wang ${ }^{1,2^{*}}$, Zhijian Chen ${ }^{1,3}$ and Lixin Xiao ${ }^{1,3^{*}}$
}

\begin{abstract}
Ferroelectric materials exhibiting anomalous photovoltaic properties are one of the foci of photovoltaic research. We review the foundations and recent progress in ferroelectric materials for photovoltaic applications, including the physics of ferroelectricity, nature of ferroelectric thin films, characteristics and underlying mechanism of the ferroelectric photovoltaic effect, solar cells based on ferroelectric materials, and other related topics. These findings have important implications for improving the efficiency of photovoltaic cells.
\end{abstract}

Keywords: solar cell, ferroelectric, photovoltaic, perovskite

\section{INTRODUCTION}

Solar energy is one of the most important sources of renewable energy. Silicon-based solar cells are among the most widely commercialized photovoltaic devices, with global installed capacity reaching $25 \mathrm{GW}$. State-of-the-art siliconbased devices have achieved an efficiency of $25.6 \%$, which is close to the theoretical limit of $\sim 30 \%$ efficiency, according to the Shockley-Queisser (S-Q) theory [1]. In addition to the efficiency limitation, the manufacturing of these cells is relatively expensive. Similar drawbacks characterize other high-efficiency single-junction cells, such as GaAs, $\mathrm{CdTe}$, and $\mathrm{CuInGaSn}$. To overcome these drawbacks, tandem structures were invented. However, these structures are complicated and very expensive. Therefore, the quest for novel types of solar cells continues.

In single-junction photovoltaic devices, photo-excited carriers are generated within an active optical absorption layer after absorbing solar photons. The generated electrons and holes then move to the corresponding electrodes, yielding a photocurrent. The photoelectric conversion mechanism prescribes that only photons with energies above the band gap energy can produce photo-generated carriers. Consequently, based on thermodynamics arguments, the open-circuit voltage $\left(V_{\mathrm{oc}}\right)$ should be lower than the band gap energy [2]. This leads to the following dilemma associated with the solar energy conversion: a narrower band gap can absorb more photons, but yields a lower voltage; on the other hand, a wider band gap can yield a higher voltage, but the current will be lower because lesser solar photons will be absorbed. Thus, the conversion efficiency appears to be limited by such contradictory concepts and techniques.

In recent years, a variety of ferroelectric photovoltaic devices have been developed. Devices based on ferroelectric materials yield a $V_{\text {oc }}$ that is much higher than the band gap of the corresponding active material owing to a strong internal electric field and the series circuit formed by the material's ferroelectric domains. This mechanism, which refers to the internal electric field formed by ferroelectric dipoles rather than traditional single-junction structures, opens new venues for designing novel photovoltaic devices. Compared with single-junction devices, ferroelectric photovoltaic devices have simpler structures and require cheaper constitutive materials. The efficiencies of these devices have been suggested to surpass the limit of the S-Q theory. The natural properties of ferroelectric materials, including their unique photoelectric conversion capability, large photo-generated voltage, and the regulation of additive electric field, bear a great promise for developing novel photovoltaic devices.

\section{CHARACTERISTICS AND MECHANISM OF THE FERROELECTRIC PHOTOVOLTAIC EFFECT}

Ferroelectrics have been the subject of research since 1920,

\footnotetext{
${ }^{1}$ State Key Laboratory for Artificial Microstructure and Mesoscopic Physics, School of Physics, Peking University, Beijing 100871, China

${ }^{2}$ Collaborative Innovation Center of Extreme Optics, Shanxi University, Taiyuan 030006, China

${ }^{3}$ Co-Innovation Center for Micro/Nano Optoelectronic Materials and Devices, Chongqing University of Arts and Sciences, Chongqing 402160, China

${ }^{*}$ Corresponding authors (emails: wangsf@pku.edu.cn (Wang S), lxxiao@pku.edu.cn (Xiao L))
} 
when French scientists discovered that Valasek Rochelle salt $\left(\mathrm{NaKCHO} \cdot 4 \mathrm{H}_{2} \mathrm{O}\right)$ exhibits ferromagnetic-like response to applied electric fields, which leads to the introduction of "ferroelectric" and "ferroelectricity" concepts [3]. Ferroelectric materials are materials that exhibit spontaneous polarization, with polarization vector direction modulated by the application of an electric field within a certain range of temperatures. Ferroelectric materials with excellent physical properties are very promising for commercial applications. Since the 1970s, with the development of processing techniques, various ferroelectric thin films (thickness ranging from a few nanometers to a few microns) with performance superior to conventional bulk materials were successfully fabricated. Up to date, thousands of types of ferroelectric crystals have been introduced. The use of ferroelectric thin films has been actively considered in photovoltaics. Ferroelectric crystals vary widely, with 10 point groups identified so far, from simple cubic to complicated monoclinic. Ferroelectrics can be categorized into double oxide ferroelectrics, nonoxide ferroelectrics, and hydrogen-bonding ferroelectrics according to the most common classification method based on their crystal chemistry. Ferroelectrics in the double oxide group, exhibiting the structural features of oxygen octahedron, are the most important. Their crystal structure is of the perovskite type, first discovered for calcium titanate oxide $\left(\mathrm{CaTiO}_{3}\right)$ compounds. Schematically, this structure can be expressed as $\mathrm{ABO}_{3}$, where $\mathrm{B}$-site ions and six oxygen ions form an octahedral structure, and A-site ions are located within the cavity between octahedrons [4].

\section{The properties of ferroelectrics}

In the presence of an external electric field, $\boldsymbol{E}$, the polarization $\boldsymbol{P}$ exhibits special nonlinear dielectric behavior. The hysteresis curve that is obtained when $\boldsymbol{P}$ is plotted $v$ s. $\boldsymbol{E}$ is very similar to the hysteresis loop of a typical ferromagnetic material. Ferroelectrics owe their name to this similarity with ferromagnetic materials [5]. Some ferroelectrics exhibit spontaneous electrical polarization in a certain range of temperatures. Such polarization can only be obtained for several directions along the crystal axis. Thus, it is different from ferromagnetic spontaneous magnetization. In ferroelectrics, the region consisting of a small number of cells with the same polarization is called ferroelectric domain. The boundary of a ferroelectric domain is called ferroelectric domain wall. A typical domain wall spans a few lattice constants. For single-crystal ferroelectrics, the relative orientation of the ferroelectric domain walls is usually $180^{\circ}$ or $90^{\circ}$, while other values are possible as well depending on the material's crystal structure. For polycrystalline ferroelectrics, different ferroelectric domain wall orientations have been observed. The dynamic process in which several domains merge to form one domain, or the process of spontaneous polarization reversal, is called domain switching. In the presence of an external electric field, the domains with polarization direction along the electric field lines expand. When all domains are polarized along the external electric field lines, the material reaches the state of saturated polarization. The saturation polarization of a ferroelectric material is usually higher than its spontaneous polarization, although the values can be comparable if the external electric field acts along the direction of spontaneous polarization.

The most typical perovskite-type ferroelectric is barium titanate $\left(\mathrm{BaTiO}_{3}, \mathrm{BTO}\right)$, whose spontaneous polarization derives mainly from the $\mathrm{Ti}^{4+}$ ion displacement. The overall energy of these materials decreases owing to the orbital hybridization of the $\mathrm{d}$ orbitals of $\mathrm{B}$ site atoms and the $\mathrm{p}$ orbital of oxygen. However, when the B-site ion is close to the oxygen atom, the elastic energy increases owing to the lattice distortion. Spontaneous displacement polarization is possible only when the energy reduction caused by a hybrid is larger than the energy gain caused by the distortion. For BTO, the reduction in the hybrid energy is larger than the energy gain owing to the lattice distortion; thus, this material exhibits spontaneous polarization. Some adjacent cells of BTO then exhibit spontaneous polarization along one of the crystal axes, while other population of cells exhibit spontaneous polarization along a different axis. A crystal phase transition from the paraelectric to ferroelectric phase occurs at the Curie temperature. This transition is characterized by the appearance of small unit cells with differently oriented spontaneous polarization, yielding ferroelectric domains [6].

Hundreds of different types of ferroelectric materials have been discovered till date, some of which had significant impact on the modern economy and technology. Ferroelectric thin films refer to ferroelectric materials with thicknesses ranging from several tens of nanometers to several micrometers. These films are widely used in microelectronics, optoelectronics, integrated optics, and micro-mechanical systems. Compared with their corresponding bulk materials, thin films of ferroelectrics show much faster response to light. With the rapid development of semiconductor industry, integration and miniaturization of electronic devices has become a new goal, with ferroelectric films receiving significant attention. Owing to innovative thin-film growing techniques and improved 
understanding of these materials, ferroelectric components have become of significant interest. Ferroelectric thin films have several unique properties, such as polarization characteristics, piezoelectric effect, pyroelectric effect, electro-optic effect, high dielectric constant, and nonlinear optical properties. Research on these properties, applications, and preparation of ferroelectric thin films is thriving, and promises to deliver novel functional materials and devices.

With the development of ferroelectric thin-film processing techniques, ferroelectric devices has developed significantly, and many new functional devices have been manufactured. The photovoltaic effect in nonpolar crystals is already well known, with crystalline silicon solar cells being the world's most widely used photovoltaic devices. However, the development of crystalline silicon solar cells is limited by the theoretical efficiency constraints. Given these constraints, significant attention has been devoted to developing novel photovoltaic materials. Ferroelectric materials with unique anomalous photovoltaic effect can yield large $V_{\text {oc }}$ for surpassing the band gap limit. An additional advantage of these materials is their relatively low fabrication cost. Owing to these advantages, ferroelectrics have been receiving increasing attention in the field of novel photovoltaic materials.

\section{The characteristics of ferroelectric photovoltaics}

The majority of ferroelectric materials exhibit both semiconductor and ferroelectric properties. A ferroelectric crystal absorbing light according to its intrinsic absorption or impurity band absorption generates an external steady state current when short-circuited. In the open-circuit state, the crystal will yield a relatively high voltage. This voltage can be 2-4 orders of magnitude higher than the band gap $\left(E_{\mathrm{g}}\right)$ of the crystal [7]. In addition, one study found that the generated voltage is proportional to the thickness of the crystal in the direction of measurement, which is therefore considered a bulk effect. This photovoltaic effect is completely different from the effect observed in nonpolar structures (such as a p-n junction or a metal-semiconductor rectifying contact). In what follows, we call it the anomalous photovoltaic (APV) effect [8].

Compared with the classical photovoltaic effect in semiconductors, some notable features of the APV effect are as follows:

(1) It is a bulk effect, appearing in a homogeneous material. It is different from the general p-n junction interface photovoltaic effect.
(2) In response to illumination, ferroelectrics can produce a stable photo-induced current or voltage along the direction of spontaneous polarization, and this effect disappears in the paraelectric phase.

(3) Light-induced voltage is proportional to the distance in the direction of an electric field, for field intensities in the $10^{3}-10^{5} \mathrm{~V} \mathrm{~cm}^{-1}$ range.

In the early 1950s, the photovoltaic effect was observed in ferroelectric ceramics. Today, it is understood that in response to illumination, ferroelectrics generate charge carriers (electrons and holes) after absorbing photons and produce photovoltaic output by forcing photo-generated electrons and holes to move to the positive and negative electrodes, respectively. This is a kind of bulk asymmetry effect that is completely different from the one observed for silicon interfacial $p-n$ junctions [9-13].

\section{The mechanism of ferroelectric photovoltaic}

In 2010, Yang et al. [14] prepared bismuth ferrite $\left(\mathrm{BiFeO}_{3}\right.$, BFO) thin films with domain walls aligned along the $71^{\circ}$ and $109^{\circ}$ directions. The BFO films with different thicknesses were grown on single-crystalline $\mathrm{DyScO}_{3}$ substrates using metal-organic chemical vapor deposition. Growth on annealed substrates resulted in ordered arrays with domain walls in the $71^{\circ}$ direction, while ordered arrays with domain walls in the $109^{\circ}$ direction were grown on un-annealed substrates. As Fig. 1 shows, when the domain walls were perpendicular to the electrodes, the $V_{\mathrm{oc}}$ reached 16 $\mathrm{V}$, and the short-circuit current $\left(J_{\mathrm{sc}}\right)$ was $1.2 \times 10 \mathrm{~mA} \mathrm{~cm}^{-2}$. The $V_{\text {oc }}$ between the electrodes increased linearly with increasing the number of domain walls and with increasing the distance between the test electrodes. In the case of the domain walls parallel to the direction of the electrodes, the $V_{\mathrm{oc}}$ and $J_{\mathrm{sc}}$ were negligible. This suggested that a large photo-voltage is not related to the material's noncentrosymmetric molecular structure, but rather may be related to its unique ferroelectric domain structure. Comparative analysis was performed for the films with domain walls aligned along the $109^{\circ}$ direction. These films yielded only a weak photo-voltage, and this was attributed to the co-existence of domains with opposite electrical polarizations. The electric fields produced by these oppositely polarized domains canceled each other out, therefore yielding no photovoltaic signal.

At the same time, Yang et al. [14,15] reported a unique phenomenon-the photovoltaic characteristic of a singledomain-structure BFO thin film did not increase significantly with varying the film thickness. In a subsequent study, the authors prepared single-crystal BFO films and 
$\mathbf{a}$
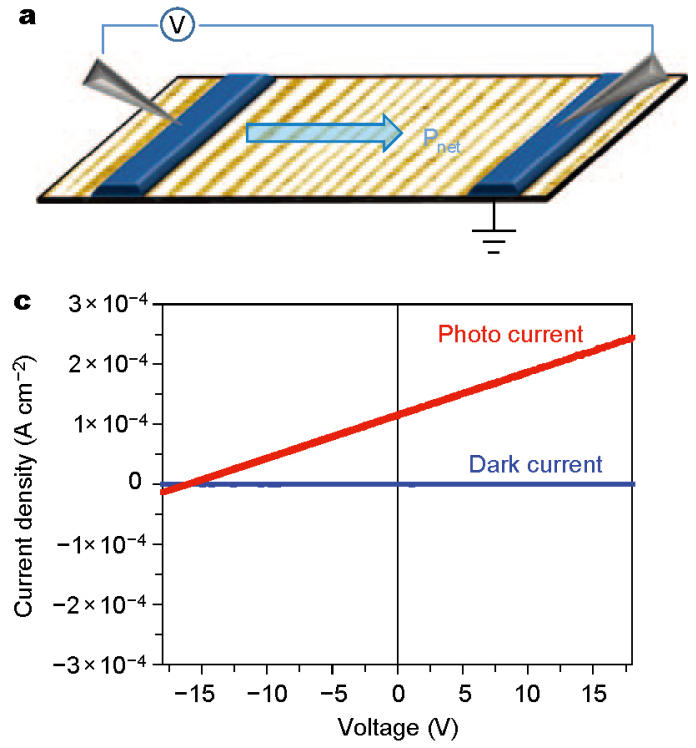
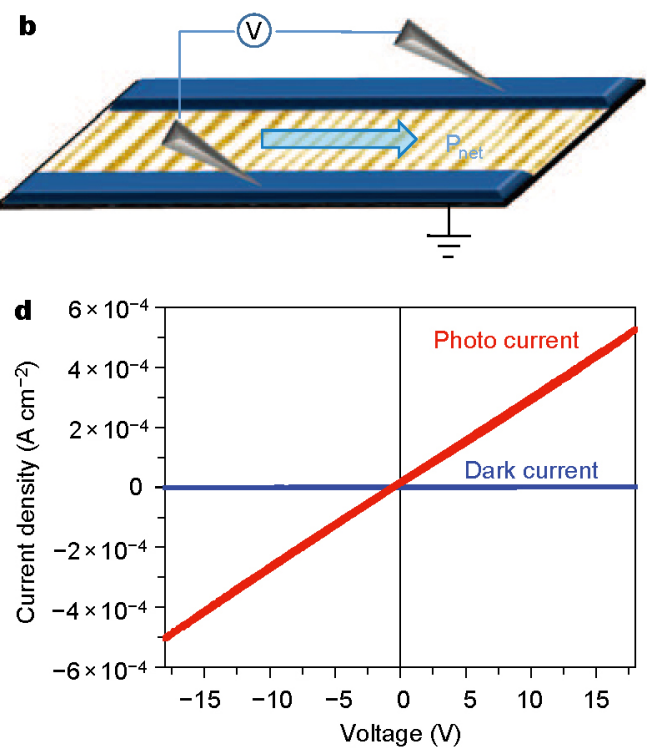

Figure 1 Schematic diagrams of (a) the perpendicular and (b) parallel devices. $I-V$ measurements of (c) the perpendicular and (d) parallel devices. Reprinted with permission from Ref. [14], Copyright 2010, Macmillan Publishers Ltd.

revealed a charge separation mechanism dramatically different from the traditional ones. This mechanism was observed for film thicknesses in the 1-2 nm range, and the obtained photo-voltage was significantly higher than the material's band gap. This voltage could be linearly extended by increasing the inter-electrode test distance, suggesting that a longer distance can yield a higher voltage. Therefore, the authors proposed a novel ferroelectric photovoltaic formation mechanism: the photovoltaic effect in the BFO thin films is caused by the $71^{\circ}$ or $109^{\circ}$ domain walls, and the bulk effect is very small. Carrier dissociation occurs at a ferroelectric domain wall because electrons move towards one side of the wall while holes move towards the other side, thereby producing photo-voltage and photocurrent. These results suggest that the photovoltaic effect in ferroelectric thin films arises owing to the ferroelectric domain periodic potential field.

Yang et al. proposed the following mechanism to explain the much stronger photovoltaic response of ferroelectrics compared with that observed in conventional silicon $\mathrm{p}-\mathrm{n}$ junctions:

(1) The internal electric field is strong. In silicon p-n junctions, the voltage associated with a $1-\mu \mathrm{m}$-thick depletion layer is $0.7 \mathrm{~V}$; thus, the internal electric field is $0.7 \mathrm{kV} \mathrm{mm}^{-1}$ [15]. In the BFO thin films, the potential difference between the domains for a typical domain wall thickness of $2 \mathrm{~nm}$ is $10 \mathrm{mV}$; thus, the internal electric field is $5 \mathrm{kV} \mathrm{mm}^{-1}$ [14].

(2) There are many ferroelectric domains in the ferro- electric materials, forming a series of circuits.

As shown in Fig. 2, Yang et al. [14] interpreted the mechanism of the ferroelectric photovoltaic response using the concept of ferroelectric domains, which is an extremely important breakthrough.

In addition, in ferroelectrics the band gap in the domain walls has been shown to be somewhat small [16]. The conductivity, which importantly affects the photovoltaic properties of ferroelectrics, is different along the domain walls. Certainly, there is an intrinsic link between the photoelectric conversion efficiency (PCE) and domain structure. Additional work needs to be done to clarify the formation mechanisms of ferroelectric photovoltaics, especially the mechanisms related to ferroelectric domains. Further research will help to shed light on the mechanisms of ferroelectric photoelectric conversion, thus helping to develop powerful solar cells based on ferroelectric materials.

\section{Advances and open issues}

Photovoltaic ferroelectric cells have become the object of intensive studies following the demonstration of the photovoltaic effect in ferroelectric thin films. In traditional silicon thin-film photovoltaic devices, electron-hole pairs are separated mainly by interfacial effects [7], such as the p-n junction, heterojunction, and Schottky barrier $[17,18]$. Devices with junction field structure need not only significantly different materials to yield a sufficiently strong electric field, but also good contact between these materials, which is a key factor determining their performance. Ow- 
a

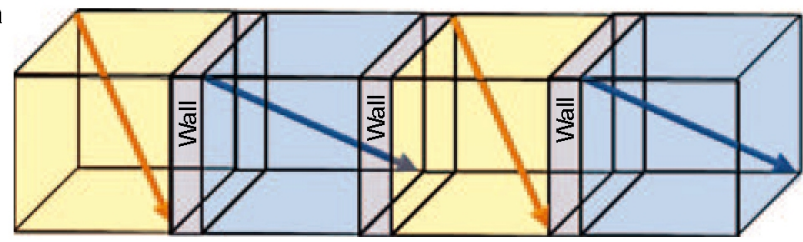

d

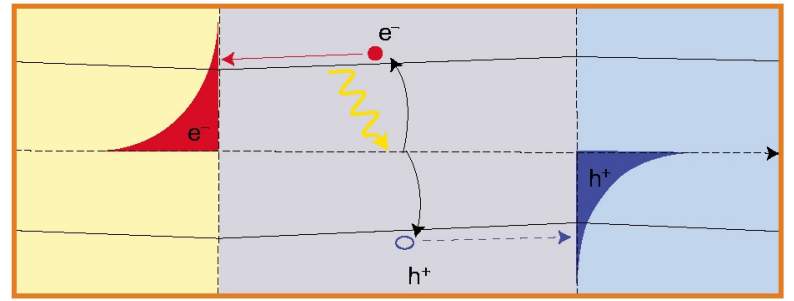

b

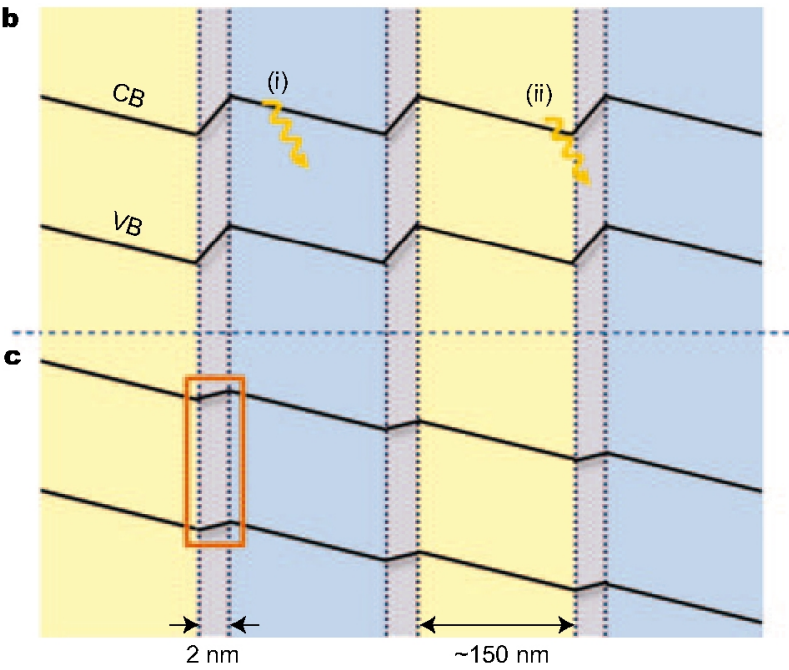

Figure 2 Schematic of domain wall mechanism, according to Yang et al. (a) Schematic of domains (the $71^{\circ}$ domain walls). (b) Band structure in the dark. (c) Band structure upon illumination. (d) Dynamics of photo-excited charges at the domain wall. Reprinted with permission from Ref. [14], Copyright 2010, Macmillan Publishers Ltd.

ing to the internal electrical polarization or the existence of domain walls with internal electric field, ferroelectric films do not require the p-n junction or Schottky barrier for realizing the separation of charge $[14,19]$. The Curie temperature of different ferroelectric materials is different; for some materials this temperature is below room temperature, for others it is close to room temperature, and still for some it is above several hundred degrees. Many ferroelectric materials with relatively high Curie temperature have been demonstrated; these materials can be used in solar cells because they can retain their ferroelectric properties even at practically high temperatures associated with the operation of solar cells. Significant research has been performed on the photovoltaic characteristics of BTO [20,21], lead zirconate titanate $\left(\mathrm{PbZr}_{x} \mathrm{Ti}_{1-x} \mathrm{O}_{3}, \mathrm{PZT}\right)$ [22-24], bismuth titanate $\left(\mathrm{Bi}_{4} \mathrm{Ti}_{3} \mathrm{O}_{12}\right)$ [25], potassium sodium niobate $\left(\mathrm{K}_{0.5} \mathrm{Na}_{0.5} \mathrm{NbO}_{3}, \mathrm{KNN}\right)[26,27]$, and BFO [21,28,29].

Techniques for fabricating ferroelectric thin films can be grouped into the following three categories: (1) physical vapor deposition (PVD), (2) chemical vapor deposition (CVD), and (3) chemical bath deposition (CSD).

PVD is usually conducted in strong vacuum. The pulsed laser deposition (PLD) and the pulsed laser ablation deposition methods belong to this category [30,31]. In this process, the studio pressure, energy and scan area of the laser beam pulse, distance and the angle of a target and a substrate, composition of the target, and other parameters can all be precisely controlled. PLD is easily controllable and yields high-quality films, thus demonstrating obvious advantages for preparation of multi-compounds.
However, using PLD, it is difficult to fabricate large-area films. At the same time, owing to the laser energy concentration, the bumping phenomenon of targets arises, leading to the formation of target debris particles on the film surface, thus significantly affecting the fabrication process and the resulting film. This phenomenon underlies the PLD's low productivity and poor shape retention. Ferroelectric thin-film deposition can be achieved by substituting the laser beam in PLD with a high-energy ion beam, and this method is known as the sputtering method (sputtering) [32]. If the energy of the ion beam is moderate, the prepared film surface will not feature target debris particles. At the same time, the advantage of accurately reproducing the target component is lost, and the deposition rate is lower. A method in which different molecular beams or atomic beams are used for directly bombarding the substrate is called molecular beam epitaxy (MBE) [33]. MBE can be used to grow films with atomic scale thickness. This technique is quite complex and requires a series of adjustments, but it is popular in laboratory research owing to its controllability, repeatability, and suitability.

A disadvantage of CVD is selecting an appropriate precursor. Deposition temperature and partial pressure of the precursor determine the feasibility and deposition rate of CVD [34]. To avoid overly complex equipment requirements, two types of precursors that are used at the same time should have similar melting points, boiling points, partial pressures, and other physical characteristics. Compared with PVD, the CVD method has three obvious advantages: (1) the ability to deposit over large areas, (2) good 
conformality, and (3) relative ease of use. This technique also has three significant drawbacks: (1) precursor toxicity and high cost, (2) a more difficult process to master, and (3) a high deposition temperature.

Among the CSD methods, the most typical and most commonly used method is polymeric solution gelation (Sol-Gel) [35,36]. This method allows to obtain a stable sol and effective control of the polycondensation reaction. Selecting adequate organometallic compounds is important, which should be characterized by a high metal content, high solubility in a solvent, free melt or vaporization during thermal decomposition, and stability at room temperature. Given these requirements, only a limited number of metallorganics can be used. Because this technique consists of several steps, problems may occur during any step, which is not conducive to the quality control of the fabricated films. The CSD reaction is conducted in solution; thus, the uniformity of the fabricated films is excellent and the stoichiometric ratio can be precisely controlled. This technique enables a precise doping component design, less equipment investment, and large area deposition. Owing to these advantages, the Sol-Gel method can potentially be used in commercial production. However, the cumbersome stepwise processing associated with the Sol-Gel method makes it difficult to precisely control the decomposition. Furthermore, the raw materials are expensive and this technique negatively impacts the environment. These disadvantages prevent the use of this technique in commercial production.

Ferroelectric materials have been considered for developing a new generation of photovoltaic devices. In 2009, Choi et al. [37] were the first to report the photovoltaic effect in BFO, which generated significant interest in ferroelectric materials for photovoltaic studies. Again, the ferroelectric photovoltaic effect was in the spotlight. Studies of ferroelectric photovoltaic devices addressed the effect of ferroelectric polarization on the photocurrent direction, ferroelectric domains in the direction of photo-voltage, the effect of indium tin oxide (ITO) or Au electrodes on light quantum efficiency, the effects of the wavelength and intensity of incident light on conversion efficiency, and the effects of the sample thickness on the photon absorption efficiency of ferroelectric materials [38-40]. Ferroelectricity (mainly polarization) directly affects the photovoltaic properties (light-generated current, voltage) of ferroelectric materials. Interfacial structure and status (including the height of the interfacial Schottky barrier, and the interfacial layer thickness) of ferroelectric thin films significantly affect their photovoltaic properties. Different inter- facial structures and states owing to different electrode configurations are likely to significantly affect the photovoltaic properties of ferroelectric thin films.

However, owing to the relatively low electrical conductivity of ferroelectric materials, the migration rate of electron-hole pairs is low; thus, the short-circuit photocurrents in most metal/ferroelectric thin film/metal (MFM) structures are low as well. The photocurrents generated in ferroelectric thin films are in general very small, on the order of $\mathrm{nA} \mathrm{cm}^{-2}$. In addition, the band gaps of conventional ferroelectric materials are relatively wide $(\sim 3.5 \mathrm{eV})$; therefore, it is difficult for these materials to absorb the entire visible spectrum. This results in low cell efficiency, similar to that of photovoltaic devices.

Typically, the photovoltaic effect consists of the following two basic processes:

(1) Electron-hole pairs are excited by the absorbed light;

(2) Internal electric field is formed by asymmetric interfaces (such as the p-n junction or the Schottky barrier) or depolarization field in ferroelectric materials for separating electron-hole pairs.

To achieve high photovoltaic efficiency, these two processes must be efficiently implemented. Photovoltaic devices are power devices; thus, the output current and output voltage are both required, because electric power is the product of voltage and current. High-performance photovoltaic devices require not only an efficient absorption of visible light and high quantum efficiency (output current), but also a sufficiently strong internal electric field (output voltage). Since the discovery of the ferroelectric photovoltaic effect, no significant breakthrough has been made in the field of ferroelectric photovoltaic devices. This is mainly owing to the difficulties of identifying ideal materials for devices and understanding the mechanisms of the ferroelectric photovoltaic effect. Both the efficiency and the mechanisms of ferroelectric-based photovoltaic devices deserve further studies.

\section{RESEARCH PROGRESS AND APPLICATIONS}

Although open-circuit photo-voltages as high as $16 \mathrm{~V}$ were achieved [14], short-circuit currents have not seen substantial improvement. Consequently, the PCE has not been significantly improved, and more experimental and theoretical studies are necessary. This issue is critical for junction-free photovoltaic devices with reasonable absorption of visible light, good photoconductivity, and strong polarization. Significant amount of research has been performed recently, aiming to achieve high-efficiency 
devices. However, these attempts have encountered some difficulties. Some new cells, such as organic trihalide perovskite (OTP)-based devices, have demonstrated efficiency improvement. Regarding single-junction devices, such as p-n junctions and Schottky junctions, the structure is usually complex, materials are relatively expensive, and fabrication is not well-controlled. These problems preclude mass fabrication of these devices. Moreover, the efficiency of these devices is limited by the S-Q limit because charge separation in these devices is determined by thermodynamic equilibrium processes. Devices based on ferroelectric photovoltaics are relatively simple; thus, the manufacturing cost and the process requirements are generally less constraining than those of single-junction devices. Moreover, the efficiency of these devices is not limited by the S-Q theory. This suggests that the ferroelectric photovoltaic effect can be expected to find use in the photovoltaic field. Other mechanisms and their combinations have been considered, such as separating charges using the depolarization field. Recently, significant amount of research has been performed on ferroelectric materials. Replacement of constituent elements and combination with other materials have been considered as well. This section will summarize the results of these studies.

\section{Improvement of material properties}

In traditional ferroelectric materials, such as PZT, BFO, $\mathrm{PbTiO}_{3}$, high band gap and low light conductivity are the main drawbacks. The most widely considered approach for alleviating the problem of high band gap is the cation substitution approach. This approach allows to reduce the band gap of the materials without affecting their ferroelectricity [41-43]. After transition metal substitutions of perovskite B-site ions, perovskite oxides can generate various semiconducting ferroelectric oxides [44]. Grinberg et al. $[45,46]$ reported a ferroelectric solid solution with a very narrow band gap of $1.39 \mathrm{eV}$. Nechache et al. [47] found that the band gap of double perovskite $\mathrm{Bi}_{2} \mathrm{FeCrO}_{6}$ (BFCO) can be adjusted in the $1.4-2.7 \mathrm{eV}$ range by varying the $\mathrm{Fe} / \mathrm{Cr}$ ratio. Using this method, these authors obtained low band gap BFCO solar cells with efficiency of $8.1 \%$. This is possible because the ferroelectricity of BFCO is decided by $\mathrm{Bi}^{3+}$ at $\mathrm{A}$ sites and the band gap is decided by the interaction between $\mathrm{Fe}$ and $\mathrm{Cr}$.

When the band gap of a ferroelectric oxide is indeed reduced using the cation substitution method, structural disorder and nonstoichiometric defects or oxygen vacancies will also be introduced into the system, which usually yields low carrier mobility and poor transport properties. Except for a few cases [47], most cation-doped semiconducting ferroelectric oxide-based devices exhibit energy conversion efficiency under $1 \%$ and photocurrent densities on the order of $\mu \mathrm{A} \mathrm{cm}^{-2}[45,48-50]$. Wang et al. [51] demonstrated a new strategy for designing stoichiometric semiconducting perovskite ferroelectric materials. This method amounts to transforming the parent nonpolar semiconducting perovskite sulfide $\mathrm{ABS}_{3}$ with Pnma symmetry into ferroelectric Ruddlesden-Popper $\mathrm{A}_{3} \mathrm{~B}_{2} \mathrm{~S}_{7}$ perovskite with spontaneous polarization. Considering that Ruddlesden-Popper $\mathrm{Ba}_{3} \mathrm{Zr}_{2} \mathrm{~S}_{7}$ has already been synthesized experimentally [52], these authors extended their investigation to Ruddlesden-Popper $\mathrm{A}_{3} \mathrm{~B}_{2} \mathrm{~S}_{7}$ sulfides derived from the parent Pnma $\mathrm{ABS}_{3}$, and studied their rotation-induced ferroelectricity. Based on their computational calculation and prediction, the Ruddlesden-Popper $\mathrm{Ca}_{3} \mathrm{Zr}_{2} \mathrm{~S}_{7}$ and other derived compounds have small band gaps $\left(E_{\mathrm{g}}<2.2 \mathrm{eV}\right)$ and exhibit stable ferroelectricity at room temperature. This work provided some guidelines to experimental synthesis of ferroelectric Ruddlesden-Popper sulfides $\mathrm{A}_{3} \mathrm{~B}_{2} \mathrm{~S}_{7}$ and fabrication of sulfide-based ferroelectric-photovoltaic devices.

The above methods allow bandgap tuning of ferroelectric materials to some extent. However, the optical conductivity of ferroelectric materials remains low, and there is no effective way to resolve this problem. The low conductivity seriously affects charge transport in these devices, which is the main reason for relatively weak photocurrents generated by these devices. In 2008, Shvydka and Karpov [53] proposed a novel photovoltaic device that used the polarized electric field formed by the polarization of a matrix of ferroelectric nanoparticles to generate the internal electric field. In this photovoltaic device, the light-absorbing material required to absorb light and generate carriers was a semiconductor, and the internal electric field required to separate the carriers was the depolarization field of the ferroelectric dipole. The field reversal between the host semiconductors and nanoparticle materials was related to the polarization interfacial charge. The dipole particles occupied a small volume fraction, and electrons and holes moved mostly through the host material. While the intra-particle field was in the direction opposite to that of the average electric current, the electric field in the host material was significantly enhanced by the dipole particles and effectively promoted the separation of the electron-hole pairs. Because the carrier transport occurred entirely in the semiconductor, there were no rigid requirements regarding the interface between the semiconductor and ferroelectric nanoparticles. This implies that fabrica- 
tion and control can be improved while the manufacturing cost can be significantly reduced. Theoretical calculations suggested that a semiconductor thin-film doped with CdS nanoparticles with volume concentration of $\sim 10 \%$ can produce a $3 \times 10^{4} \mathrm{~V} \mathrm{~cm}^{-1}$ internal electric field, which is comparable to that of a typical p-n junction. Therefore, the internal electric field required to separate the carriers can be provided by doping ferroelectric nanoparticles. Therefore, such a ferroelectric-semiconductor-coupled device is promising for obtaining reasonable photo-voltage and photocurrent output.

In 2009, Jha et al. [54] produced a device with the structure of glass/transparent conducting oxide (TCO)/CdTe$\mathrm{CdS} /$ electrode using the vacuum coating technology. The efficiency of this device reached $8.3 \%$, and the average efficiency was above $6 \%$, while the $V_{\text {oc }}$ was above $0.6 \mathrm{~V}$. The average $V_{\text {oc }}$ of pure CdTe devices (glass/TCO/CdTe/metal electrode) fabricated using the same processes was below $0.46 \mathrm{~V}$, and the maximum $V_{\text {oc }}$ was below $0.6 \mathrm{~V}$. This difference between the two samples shows that the introduction of CdS nanoparticles is feasible for enhancing the performance of photovoltaic devices. As shown in Fig. 3, Huang et al. $[55,56]$ increased the efficiency of a CdS ferroelectric-semiconductor coupled cell to $8.8 \%$, and studied micro and macro mechanisms of this device. In subsequent studies, they increased the efficiency of this photovoltaic device to $11.3 \%$, and reported the average efficiency of $11 \%$. Many nanoparticle materials with strong dipole moment are available today [57-59]. Ferroelectric-semiconductor coupled photovoltaic devices can possibly be designed and fabricated by combining nanoparticles and light-absorbing

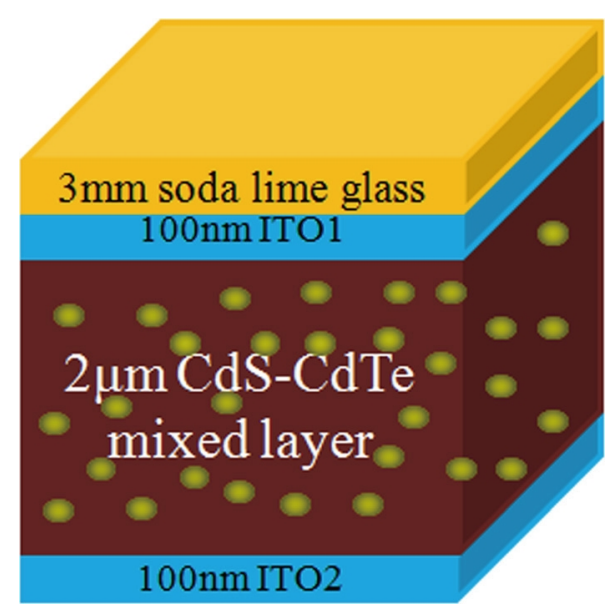

Figure 3 Schematic of the CdS nanodipole solar cell by Huang et al., reprinted with permission from Ref. [55], Copyright 2013, AIP Publishing. materials. Therefore, the ferroelectric-semiconductor coupling method is certainly feasible for ferroelectric photovoltaic research.

\section{Combination with various materials}

Given the low photoconductivity limitation and the wide band gap of ferroelectrics, it is clear that these drawbacks cannot be alleviated by using pure ferroelectrics. Thus, combination ferroelectrics with other materials and designing devices based on these combinations became an important research venue. Various setups have been considered, such as multilayer structures consisting of ferroelectric materials and semiconductor materials [34,60-66].

Migration rate of electron-hole pairs in ferroelectric materials is relatively low, resulting in low short-circuit photocurrents for most MFM structures. Some semiconductor materials can effectively absorb photons and generate large numbers of electron-hole pairs. For example, metal/semiconductor/metal (MSM) structures with Ohmic contacts exhibit large photocurrents, but their $V_{\mathrm{oc}}$ is almost zero, owing to the absence of internal field for separating the electron-hole pairs [66]. In light of the above-mentioned limitations, the conversion efficiency of conventional simple MFM and MSM structures cannot meet the requirements imposed by practical applications. For a simple ITO/PZT/Pt structure, the device consists of two back-to-back Schottky diodes, and the work function of the Pt electrode is high $(5.6 \mathrm{eV})$ so that most of the electronics cannot cross into the interface Schottky barrier of the Pt electrode, and it is difficult to increase the current [22]. When studying the photovoltaic effect in $\mathrm{ITO} / \mathrm{ZnO} / \mathrm{BFO} / \mathrm{Pt}$ heterostructures, Fan et al. [67] discovered that the $\mathrm{ZnO} / \mathrm{BFO}$ interface can form an $\mathrm{n}^{+}-\mathrm{n}$ junction. A substantial increase in the photocurrent can be achieved by taking advantage of good light response properties of ZnO. PZT is a typical ferroelectric material with strong residual polarization and band gap of $3.6 \mathrm{eV}$. $\mathrm{ZnO}$ is an n-type semiconductor, which can be easily crystallized and exhibits strong light response, high electron mobility, and low recombination rate loss. $\mathrm{ZnO}$ is a good candidate material, and its band gap is $3.26 \mathrm{eV}$ lower than that of the PZT for the semiconductor layer. $\mathrm{Cu}_{2} \mathrm{O}$, with its smaller band gap $(\sim 2.1 \mathrm{eV})$, is very appropriate as a light-absorbing translucent layer. It can be used for increasing the device's photocurrent by taking advantage of the light response of $\mathrm{ZnO}$ and using the PZT/ZnO interface instead of the $\mathrm{PZT} / \mathrm{Pt}$ interface. Inspired by this idea, Zheng et al. $[63,64]$ designed a metal/semiconductor/ferroelectric/metal 
heterostructure and prepared ITO/PZT/ZnO/Pt and $\mathrm{ITO} / \mathrm{PZT} / \mathrm{ZnO} / \mathrm{Cu}_{2} \mathrm{O} / \mathrm{Pt}$ heterostructure-based thin-film devices on the basis of the ITO/PZT/Pt structure, using the magnetron sputtering method. Studies on the photovoltaic effect in $\mathrm{ITO} / \mathrm{BFO} / \mathrm{ZnO} / \mathrm{Pt}$ heterostructures demonstrated that a $J_{\mathrm{sc}}$ of $340 \mu \mathrm{A} \mathrm{cm} \mathrm{cm}^{-2}$ and a PCE of $0.33 \%$ can be obtained, which is two orders of magnitude higher than the values obtained for ITO/BFO/Pt structures. In addition, $\mathrm{ITO} / \mathrm{PZT} / \mathrm{ZnO} / \mathrm{Cu}_{2} \mathrm{O} / \mathrm{Pt}$ structures give full consideration to the reasonable distribution of the band gap, and can effectively promote the flow of charge. According to the effects of the layer thickness and preparation process of $\mathrm{ZnO}$ and $\mathrm{Cu}_{2} \mathrm{O}$ layers on the performance of the resulting photovoltaic devices, the $J_{\text {sc }}$ of a device that was prepared in optimal conditions was $\sim 60$-fold higher compared with that of the ITO/PZT/Pt structure.

To address the interface effect of ferroelectric materials and metal oxide semiconductors, Pan et al. [68] added a $\mathrm{ZnO}$ layer to the ITO/PZT/Au structure by preparing the samples on commercial ITO-coated glass substrates, using a sol-gel process and spin-coating technique. The performance of these devices was significantly improved compared with that of the original ones. Owing to the irreversible spontaneous polarization of $\mathrm{ZnO}$, the $\mathrm{ITO} / \mathrm{PZT} / \mathrm{ZnO} / \mathrm{Au}$ film forms a $\mathrm{p}-\mathrm{n}$ junction with polarization dependent on the strong interfacial coupling effect at the PZT/ZnO interface. This interface coupling effect underlies the depletion layer and the internal electric field, and changes the energy level structure of the heterojunction. In the case of the optimal $\mathrm{ZnO}$ layer thickness of $60 \mathrm{~nm}$, the light conversion efficiency of the device increased by two orders of magnitude. In addition, the researchers compared the performances of $\mathrm{ITO} / \mathrm{ZnO} / \mathrm{PZT} / \mathrm{Au}$, ITO/PZT/ZnO/Au and ITO/PZT/Au structures. Changing the location of the $\mathrm{ZnO}$ layer did not affect the performance of the photovoltaic device. Because the band gap of PZT is wider than that of $\mathrm{ZnO}$, the photovoltaic performance of the $\mathrm{ITO} / \mathrm{ZnO} / \mathrm{PZT} / \mathrm{Au}$ structure is similar to that of the ITO/PZT/ZnO/Au structure, indicating that the performance of the device is mainly derived from the presence of $\mathrm{ZnO}$ layers that yield more light-excited carriers. Progress has been made also in studies of multilayer film structures containing ferroelectric materials and metal oxides. As Fig. 4 shows, Chatterjee et al. [69] prepared $\mathrm{p}-\mathrm{i}-\mathrm{n}$ heterojunctions with $\mathrm{BFO}$ perovskite nanoparticles and p/n-type oxides $\left(\mathrm{NiO}-\mathrm{BiFeO}_{3}-\mathrm{ZnO}\right)$ with the $V_{\text {oc }}$ of $0.42 \mathrm{~V}$ and short-circuit current of 0.57 $\mathrm{mA}$. To fabricate the devices, a layer of $\mathrm{NiO}$ nanoparticles (serving as a p-type hole collecting material), a layer of $\mathrm{BiFeO}_{3}$ nanoparticles (serving as an absorber), and a layer of $\mathrm{ZnO}$ n-type (serving as an electron-collecting material) were formed in sequence. Compared with devices that feature only one heterojunction, the fabricated p-i-n structure device exhibited better performance.

Some studies addressed combinations of different ferroelectric materials [70-75]. Because various ferroelectric materials have different ferroelectric properties, light absorption properties, and photoconductivities combining different ferroelectric materials can not only yield efficient light absorption, but also enhance carrier charge separation through the interfacial Schottky barrier, which is of great importance for enhancing the performance of the device. Chakrabartty et al. [73] combined BFO and $\mathrm{BiMnO}_{3}(\mathrm{BMO})$ by growing $\mathrm{BFO} / \mathrm{BMO}$ bilayer thin films sandwiched between ITO top electrodes and $\mathrm{Nb}(0.5 \%)$ doped $\mathrm{SrTiO}_{3}$ (NSTO) substrates. The fabricated device exhibited the PCE of $\sim 1.43 \%$. Ferroelectric bilayers were prepared on a pre-polarized low-bandgap substrate. The energy distribution of the materials forming the ferroelectric-semiconductor interfacial Schottky barrier in the
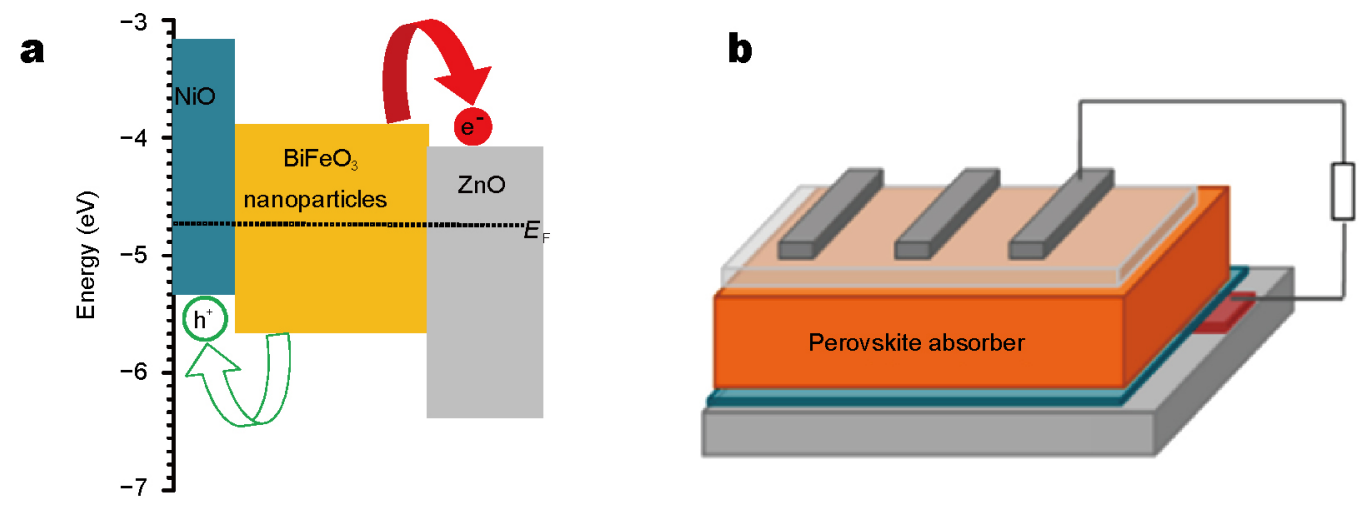

Figure 4 Schematic of the energy level diagram and device by Chatterjee et al., adapted with permission from Ref. [69], Copyright 2014, American Chemical Society. 
device enhanced the photo-excited carrier separation. BFO has strong ferroelectricity at room temperature with the band gap in the 2.3-2.8 eV range. Compared with $\mathrm{LiNbO}_{3}$, $\mathrm{BaTiO}_{3}$, or $\mathrm{Pb}(\mathrm{Zr}, \mathrm{Ti}) \mathrm{O}_{3}, \mathrm{BFO}$ has a relatively narrow band gap. BFO-based photovoltaic devices demonstrate small photocurrent density of $\sim \mathrm{nA} \mathrm{cm}{ }^{-2}$, owing to the low conductivity and wide band gap. BMO with weak ferroelectricity and a band gap of $\sim 1.2 \mathrm{eV}$ has high light absorption efficiency. For both materials, the respective photovoltaic devices exhibited PCE under 0.3\% [76,77], which limited their application. Combining the ferroelectricity of BFO and the low band gap of BMO, many devices with relatively high efficiency can be fabricated. Along this line of reasoning, Sharma et al. [72] prepared a multilayered ferroelectric $\mathrm{BFO} / \mathrm{BTO} / \mathrm{BFO} / \mathrm{BTO}$ thin-film structure deposited on a $\mathrm{Pt} / \mathrm{Ti} / \mathrm{SiO}_{2} / \mathrm{Si}$ substrate. The energy band gap of this structure was $2.62 \mathrm{eV}$, while $V_{\text {oc }}$ and $J_{\text {sc }}$ were $1.43 \mathrm{~V}$ and $12.65 \mu \mathrm{A} \mathrm{cm}^{-2}$, respectively.

The above approach of combining ferroelectric materials with different properties has achieved some progress. It is conceivable that ferroelectric materials can also be combined with other high-performance materials, such as perovskites [78-82]. Zhao et al. [1] fabricated a $\mathrm{BiFeO}_{3}$ (BFO) polycrystalline film modified by organolead trihalide perovskite $\mathrm{CH}_{3} \mathrm{NH}_{3} \mathrm{PbI}_{3}$. This device, with $J_{\mathrm{sc}}$ of 1.74 $\mathrm{mA} \mathrm{cm}{ }^{-2}$ and $V_{\text {oc }}$ of $1.62 \mathrm{~V}$, exhibited much higher performance than the original BFO device. The BFO layer in the device reached the thickness of $200 \mathrm{~nm}$ after repeated spin-coating annealing, and the perovskite layer was deposited on BFO. Because the two materials have similar lattice structures, the BFO layer affected the orientation of the perovskite layer. Perovskite is a noncentrosymmetric material, whose crystal lattice orientation is likely to yield spontaneous polarization in materials. Thus, the observed ultrahigh voltage output was attributed to the perovskite spontaneous polarization induced by the BFO lattice and the modified BFO surface work function. In that study, the BFO layer was relatively thick, and the overall conductivity of the device was reduced. This may explain why $J_{\mathrm{sc}}$ was nearly one order of magnitude lower than that of normal perovskite-based devices.

$\mathrm{Hu}$ et al. [83] prepared organic-inorganic perovskite solar cells with incorporated three-dimensional (3D) self-branching anatase $\mathrm{TiO}_{2}$ nanorod/SrTiO${ }_{3}$ nanocomposites (TS). The prepared 3D self-branching anatase $\mathrm{TiO}_{2}$ nanorod $/ \mathrm{SrTiO}_{3}$ films were coated with $\mathrm{PbI}_{2}$, followed by heating. After the samples were cooled to room temperature, $\mathrm{CH}_{3} \mathrm{NH}_{3} \mathrm{I}$ solution in 2-propanol was dropped on the $\mathrm{PbI}_{2}$-coated substrate. These authors obtained the PCE of $9.99 \%, J_{\mathrm{sc}}$ of $19.48 \mathrm{~mA} \mathrm{~cm}{ }^{-2}$, and $V_{\mathrm{oc}}$ of $\sim 0.95 \mathrm{~V}$. They compared the performances of three devices, of which the other two devices with the same thickness of $400 \mathrm{~nm}$ differed in terms of their electron transfer layers into the $\mathrm{SrTiO}_{3}$ layer (S) and $\mathrm{P} 25 \mathrm{TiO}_{2}$ layer $(\mathrm{T})$. By comparing the devices in terms of their morphology and electrical measurements, it was found that the perovskite in the T-S type device with the best PV performance has the best crystallinity. It is worth noting that compared with the conventional perovskite device $(\mathrm{T}), V_{\text {oc }}$ of the device with the STO layer added increased from 0.8 to $0.9 \mathrm{~V}$, but its $J_{\mathrm{sc}}$ decreased from $\sim 16$ to $\sim 13 \mathrm{~mA} \mathrm{~cm}^{-2}$. The devices with the $\mathrm{TiO}_{2}$ nanorod/BFO nanoparticle layer maintained higher voltage and achieved the $J_{\mathrm{sc}}$ of $\sim 19.5 \mathrm{~mA} \mathrm{~cm}^{-2}$. Zhao et al. [1] managed to increase $V_{\text {oc }}$ up to $\sim 1.6 \mathrm{~V}$ by adding an BFO layer $(200 \mathrm{~nm})$, but $J_{\text {sc }}$ remained as low as $\sim 1.7 \mathrm{~mA}$ $\mathrm{cm}^{-2}$. These results demonstrate that adding a ferroelectric layer can reduce $J_{\mathrm{sc}}$ to a different extent. Therefore, more in-depth research is needed to clearly understand the benefits and prospects of combining ferroelectric materials with OTP.

\section{The ferroelectricity of OTP}

The novel perovskite solar cells based on OTP as a light-absorbing material have been rapidly developed in recent years. The PCE of these cells increased from 3.8\% in 2009 to $20.1 \%$ in 2014 , and these devices received a great deal of attention. The high performance of perovskite solar cells is usually attributed to the good optoelectronic properties of OTP materials. In perovskite solar cells, the most widely used light-absorbing material, $\mathrm{CH}_{3} \mathrm{NH}_{3} \mathrm{PbI}_{3}$, is a direct band gap semiconductor with a band gap of $\sim 1.5 \mathrm{eV}$, which is extremely close to the optimal band gap required in a single-junction solar cell. The extinction coefficient reaches $10^{5} \mathrm{~cm}^{-1}$, and theoretically a $400-\mathrm{nm}$-thick absorbent layer can absorb all visible light [79]. In $\mathrm{CH}_{3} \mathrm{NH}_{3} \mathrm{PbI}_{3}$ the electron and hole diffusion length is longer than $100 \mathrm{~nm}$ and can reach $1 \mu \mathrm{m}$ by doping with $\mathrm{Cl}$ [80]. These advantages make OTP materials promising as absorbing materials for solar cells.

Using first-principles theoretical calculations, Frost et al. [84] obtained the rotation barrier of organic-cation spontaneous polarization and the intensity of spontaneous polarization in materials. They confirmed that ferroelectric properties exist in OTP materials. By performing firstprinciples calculations, combining the classic point charge model and the Berry phase method, Fan et al. [85] obtained consistent polarization of $\sim 8 \mu \mathrm{C} \mathrm{cm}^{-2}$. This result suggests that a ferroelectric structure of $\sim 8 \mu \mathrm{C} \mathrm{cm}^{-2}$ in the mate- 
rial is the most stable. In 2015, Filippetti et al. [86], using the density functional theory and the local density approximation, obtained the polarization of cubic $\mathrm{CH}_{3} \mathrm{NH}_{3} \mathrm{PbI}_{3}$ as $\sim 3 \mu \mathrm{C} \mathrm{cm}^{-2}$. The extreme polarization orientation was random. The authors proposed that the polarization reversal can only occur at $50-60 \mathrm{~K}$ in the presence of an external electric field. This implies that the system can exhibit local electrical polarization, but overall, it is not ferroelectric. However, the first-principles theory calculation for ferroelectric behavior of OTP is likely to contain errors, because such first-principles calculation of ferroelectric behavior requires the material to be an insulator, while OTP is a semiconductor. Although these theoretical calculations have yielded some positive conclusions, they do not confirm that OTP is ferroelectric.

Experimental studies on ferroelectrics yielded mixed results. Stoumpos et al. [87] demonstrated that $\mathrm{CH}_{3} \mathrm{NH}_{3} \mathrm{PbI}_{3}$ exhibits ferroelectric behavior based on the electric polarization (EP) loop measurements; using piezoresponse force microscopy (PFM) measurements. Kutes et al. [88] discovered ferroelectric domains and demonstrated reversible ferroelectric domain inversion. Wei et al. [89], using X-ray diffraction and EP loop measurements confirmed the existence of ferroelectricity in $\mathrm{CH}_{3} \mathrm{NH}_{3} \mathrm{PbI}_{3-x} \mathrm{Cl}_{x}$. The experimental results of Wang et al. [90] suggest the presence of ferroelectric domains, and these ferroelectric domains are completely reversible. Studying spontaneous polarization in $\mathrm{CH}_{3} \mathrm{NH}_{3} \mathrm{PbI}_{3}$ film, Zhao et al. [91] found, in PFM experiments, that $\mathrm{CH}_{3} \mathrm{NH}_{3} \mathrm{PbI}_{3}$ films exhibit uniform spontaneous polarization and the polarization direction could be reversed by applying a voltage. This was attributed to the noncenter cubic lattice symmetry and lone pairs of electrons in $\mathrm{N}^{3+}$ and $\mathrm{Pb}^{2+}$ ions. The material in the studies by Stoumpos, Kutes, and Zhao et al. was $\mathrm{CH}_{3} \mathrm{NH}_{3} \mathrm{PbI}_{3}$, while Wei studied $\mathrm{CH}_{3} \mathrm{NH}_{3} \mathrm{PbI}_{3-x} \mathrm{Cl}_{x}$, but all studies converged by concluding that lead halide perovskite is ferroelectric.

On the other hand, Xiao et al. [92] did not observe ferroelectric polarization behavior in $\mathrm{CH}_{3} \mathrm{NH}_{3} \mathrm{PbI}_{3}$, using Precision Premier II from Radiant Technologies and PFM measurements. Fan et al. [85] concluded that OTP does not exhibit ferroelectricity based on the EP loop and PFM measurements. Beilsten et al. [93] studied the conductivity hysteresis of $\mathrm{CH}_{3} \mathrm{NH}_{3} \mathrm{PbI}_{3}$ films using the double-wave method and obtained results that excluded the impact of the leakage current. They obtained a hysteresis curve consistent with that of conventional ferroelectrics. However, this hysteresis strongly depended on frequency in the $0.1-150 \mathrm{~Hz}$ range, and at frequencies under $0.4 \mathrm{~Hz}$ the charge density was 1000 $\mu \mathrm{C} \mathrm{cm}^{-2}$. This suggests that the hysteresis was owing to the motion of ions that occurred on the timescale of a second. According to the $1 / \mathrm{f}$ dependence of the charge density hysteresis, ferroelectric response stronger than $1 \mu \mathrm{C} \mathrm{cm}^{-1} \mathrm{can}$ be excluded. All of these studies suggest that $\mathrm{CH}_{3} \mathrm{NH}_{3} \mathrm{PbI}_{3}$ films are essentially not ferroelectric.

There is no uniform interpretation of the PFM results using the same standards, and other factors cannot be dismissed. Because the phase difference in PFM can be caused by interfacial modification, the local dielectric constant changes, and the motion of ions. However, the PFM experiments did not discuss related issues. There is a systematic error associated with the use of the P-E loop in the studies of OTP ferroelectricity, because P-E loops are relevant only for insulating materials. In the case of semiconductors, leakage errors and ion motion in lead halide perovskites are likely to affect the loop performance. First-principles ferroelectricity calculations are also fundamentally flawed for the same reason-these calculations assume insulators, whereas OTP is not an insulator.

When it was discovered that OTP may have ferroelectric properties, some research was performed to explore the use of ferroelectrics for improving performance. Although $V_{\text {oc }}$ excessing the material band gap was not obtained as expected, the positive effect of ferroelectric domains on photo-generated carriers was found [84,94-96]. It has been proposed that the OTP ferroelectricity underlies the superior performance of perovskite devices because ferroelectric domain walls in these materials form separated migration channels for electrons and holes. As Fig. 5a shows, Frost et al. [84] put forward a migration model in which photo-generated electrons and holes were transferred via separate transmission channels formed by ferroelectric domain walls. Owing to their positive and negative charges, the electrons and holes tended to flow via different domain walls channels. According to this model, dedicated migration paths (for electrons and holes) were formed between the two electrodes. Liu et al. [97], using density functional theory (DFT) calculations, studied the energy and electrical structure of $\operatorname{MAPbX}_{3}\left(\mathrm{MA}=\mathrm{CH}_{3} \mathrm{NH}_{3}, \mathrm{X}=\mathrm{Cl}, \mathrm{Br}, \mathrm{I}\right)$ with charged and uncharged domain walls (as shown in Fig. 5b). They found that the band gap of $\mathrm{MAPbX}_{3}$ with charged domain walls is somewhat narrower, and that of $\mathrm{MAPbX}_{3}$ with uncharged domain walls is not affected.

As Figs $5 \mathrm{c}$ and d show, Sherkar et al. [96] investigated whether ferroelectric polarization can explain the superior performance of perovskite-based battery. It was proposed that the existence of ferroelectric domains does not play a significant role in the enhancement of $V_{\mathrm{oc}}$, but ferroelectric domains can form effective charge transport channels, 
a
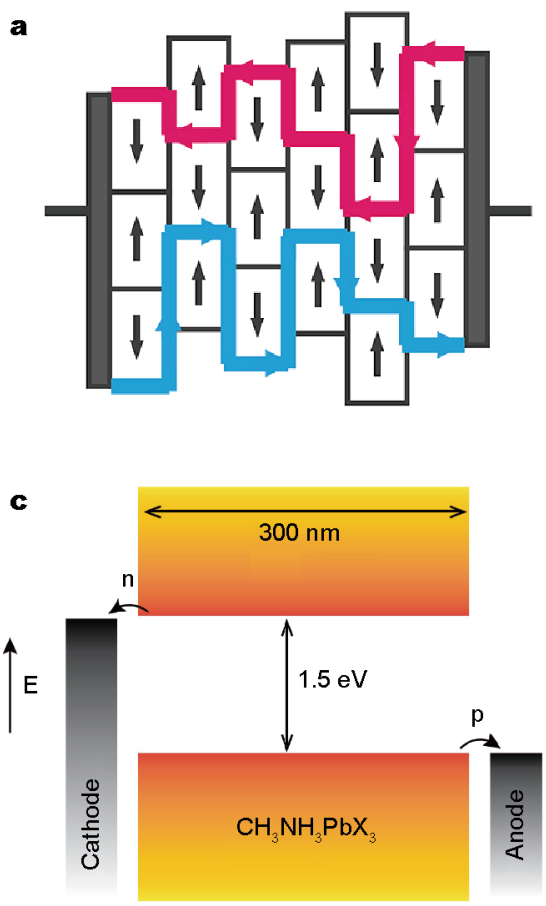

b
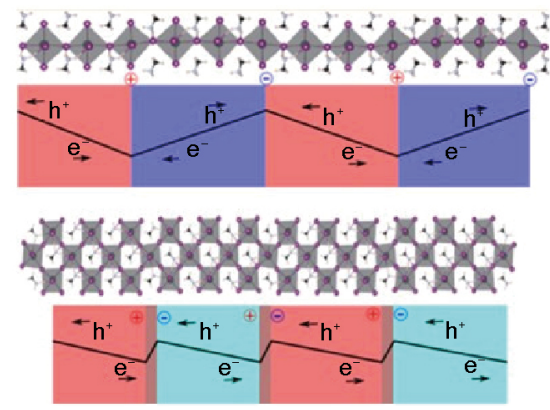

d

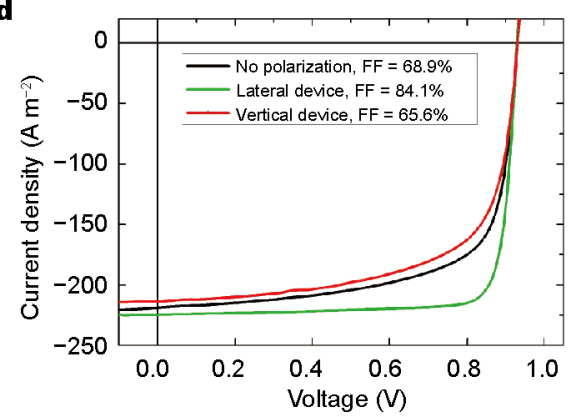

Figure 5 Schematic of the superior performance of perovskite devices derived from the ferroelectricity in OTP. (a) Migration model with separated transmission channels formed by ferroelectric domain walls, from the study by Frost et al., reprinted with permission from Ref. [84], Copyright 2014, American Chemical Society. (b) Calculated energy and electrical structure of OTP, with charged and uncharged domain wall, from the study by Liu et al., reprinted with permission from Ref. [97], Copyright 2015, American Chemical Society. (c) Device structure of the simulated perovskite solar cell and (d) simulated $J-V$ curves for this device with no polarization, vertical device, and lateral device, from the study by Sherkar et al., reprinted with permission from Ref. [96], Copyright 2016, The Royal Society of Chemistry.

reducing the carrier recombination rate. These authors designed a 3D drift-diffusion model to study the generation, transportation, and recombination of charges. For determining the relationship between the low combined rate and the ferroelectric polarization, the impact of ferroelectricity was introduced as a charged domain wall. The results showed that, for highly ordered structures with polarized ferroelectric domains, carrier transportation and recombination are strongly correlated with the size and orientation of ferroelectric domain walls. When ferroelectric domain walls were parallel to the pole of the situation, the fill factor was very low owing to the high combination rate, while $V_{\text {oc }}$ increased only slightly. When domain walls were perpendicular to the electrode, the fill factor and the $J_{\text {sc }}$ were large. This occurred owing to the low combination rate, which in turn occurred because electrons and holes traveled via separate domain wall channels towards the electrodes. For structures with randomly oriented ferroelectric domains, the effective charge transportation implied the presence of effective charge transport channels in the device, resulting in low charge carrier recombination rate. These results are consistent with the model proposed by Frost et al. [84].

No studies so far have addressed a possible effect of ferroelectric property on the carrier dynamics in OTP. However, related research has been conducted for BFO, which is one of the most promising ferroelectric materials. Yamada et al. [98] studied the optical response and dynamical behavior of photo-carriers in $\mathrm{BiFeO}_{3}$ thin films by performing transient absorption (TA) and photocurrent (PC) measurements. Fast (1 ns) and slow (100 ns) components of TA and PC decay dynamics were observed, and they were attributed to the localization of free carriers to shallow trap states and the recombination of trapped carriers, respectively. These long-lived carriers achieved a high carrier density when the carriers were continuously photo-generated. The long carrier lifetime was attributed to the ferroelectricity of $\mathrm{BiFeO}_{3}$. The depolarized field in ferroelectric $\mathrm{BiFeO}_{3}$, which reduces the photo-carrier recombination rates, spatially separates the photo-generated electrons and holes. The large associated electric field at ferroelectric domain walls can cause more effective charge separation. This research suggests that photo-carriers driven by ferroelectricity would appear to be responsible for the unique photo-response characteristics of ferroelectric semiconductors. Although ferroelectricity of OTP remains to be studied and confirmed, these theories de- 
serve attention and more in-depth studying. It is possible for OTP to play a significant role in promoting device performance in view of the photoelectric properties and potential ferroelectricity.

\section{SUMMARY AND OUTLOOK}

This article provided an overview of ferroelectricity and ferroelectric materials, including the relevant phenomena and mechanisms. The studies on ferroelectric photovoltaic devices and applications were also summarized. Improvement of material properties, achieved for example by combining different materials, is a new research direction. Ferroelectric photovoltaic devices with unique properties, such as the internal electric field induced by the depolarization field, are of great value in the field of solar energy conversion. The anomalous photovoltaic effect in ferroelectric thin films makes these devices promising. Some research on the characteristics and mechanisms of ferroelectric thin-film photovoltaics has been conducted up to date, and some progress has been made, but much work remains to be done before ferroelectric thin films can be used in solar cells.

Since the discovery of the ferroelectric photovoltaic effect, the PCE of ferroelectric devices has been limited by material properties such as carrier mobility and optical band gap. Modification of ferroelectric materials was not quite successful and introduced side effects. These drawbacks preclude a massive use of ferroelectric photovoltaic materials. A breakthrough is expected by introducing semiconductors doped with ferroelectric nanoparticles, which combines the advantages of semiconductors and ferroelectric properties. Furthermore, as a semiconductor material, OTP is likely to exhibit ferroelectric behavior and good photovoltaic characteristics. However, such ferroelectricity has not yet been precisely understood. No research has addressed the possible effect of the ferroelectric property on the carrier dynamics. More research is needed to explore the contribution of ferroelectricity to the OTP's excellent photovoltaic characteristics and photo-physics. Future research will focus on demonstrating and characterizing ferroelectricity in OTP. The mechanism of ferroelectricity as well as its effect on performance needs to be studied.

Received 1 August 2016; accepted 19 September 2016; published online 19 October 2016

1 Zhao P, Bian L, Wang L, et al. Enhanced open voltage of $\mathrm{BiFeO}_{3}$ polycrystalline film by surface modification of organolead halide perovskite. Appl Phys Lett, 2014, 105: 013901
2 Glunz SW, Feldmann F, Richter A, et al. The irresistible charm of a simple current flow pattern-25\% with a solar cell featuring a full-area back contact. 31st European Photovoltaic Solar Energy Conference and Exhibition, Hamburg, Germany, 2015: 259-263

3 Ding JN, Chen MJ, Qiu JH, et al. Photovoltaic properties of ferroelectric solar cells based on polycrystalline $\mathrm{BiFeO}_{3}$ films sputtered on indium tin oxide substrates. Sci China-Phys Mech Astron, 2015, 58: $1-6$

4 Jiang GL, Chen WJ, Zheng Y. Simulation study of domain structure evolution in ferroelectric thin film under bending mechanical loads. Sci Sin-Phys Mech Astron, 2016, 46: 044613

5 Zhang Y. Recent developments related to multifunctional ferroelectric for room-temperature applications. Sci China Technol Sci, 2016, 59: 513-514

6 Si WZ, Huang KK, Wu XF, et al. Epitaxial thin film of $\mathrm{SmFeO}_{3}$ ferroelectric heterostructures. Sci China Chem, 2014, 57: 803-806

7 Brody PS, Crowne F. Mechanism for the high voltage photovoltaic effect in ceramic ferroelectrics. J Electron Mater, 1975, 4: 955-971

8 Shockley W, Queisser HJ. Detailed balance limit of efficiency of p-n junction solar cells. J Appl Phys, 1961, 32: 510-519

9 Zheng F, Xu J, Fang L, et al. Separation of the Schottky barrier and polarization effects on the photocurrent of Pt sandwiched $\mathrm{Pb}\left(\mathrm{Zr}_{0.20} \mathrm{Ti}_{0.80}\right) \mathrm{O}_{3}$ films. Appl Phys Lett, 2008, 93: 172101

10 Qin M, Yao K, Liang YC. Photovoltaic mechanisms in ferroelectric thin films with the effects of the electrodes and interfaces. Appl Phys Lett, 2009, 95: 022912

11 Qin M, Yao K, Liang YC. High efficient photovoltaics in nanoscaled ferroelectric thin films. Appl Phys Lett, 2008, 93: 122904

12 Grekov AA, Spitsyna MA, Spitzina VD, Fridkin VM. Photoferroelectric effects in ferroelectric semiconductors of AV-type BVI-type CVII-type with low-temperature phase changes. Sov Phys Crystallogr, 1970, 15: 423-429

13 Volk T, Grekov A, Kosonogov N, et al. Influence of illumination on the domain structure and curie temperature of $\mathrm{BaTiO}_{3}$. Sov Phys-Solid State, 1973, 14: 2740-2743

14 Yang SY, Seidel J, Byrnes SJ, et al. Above-bandgap voltages from ferroelectric photovoltaic devices. Nat Nanotech, 2010, 5: 143-147

15 Yang SY, Martin LW, Byrnes SJ, et al. Photovoltaic effects in $\mathrm{BiFeO}_{3}$. Appl Phys Lett, 2009, 95: 062909

16 Qin M, Yao K, Liang YC. Photovoltaic characteristics in polycrystalline and epitaxial $\left(\mathrm{Pb}_{0.97} \mathrm{La}_{0.03}\right)\left(\mathrm{Zr}_{0.52} \mathrm{Ti}_{0.48}\right) \mathrm{O}_{3}$ ferroelectric thin films sandwiched between different top and bottom electrodes. J Appl Phys, 2009, 105: 061624

17 Li D, Wang L, Li D, et al. Formation of nanostructured emitter for silicon solar cells using catalytic silver nanoparticles. Appl Surface Sci, 2013, 264: 621-624

18 Dimitrov DZ, Du CH. Crystalline silicon solar cells with micro/nano texture. Appl Surface Sci, 2013, 266: 1-4

19 Seidel J, Fu D, Yang SY, et al. Efficient photovoltaic current generation at ferroelectric domain walls. Phys Rev Lett, 2011, 107: 126805

20 Won CJ, Park YA, Lee KD, et al. Diode and photocurrent effect in ferroelectric $\mathrm{BaTiO}_{3-\delta}$. J Appl Phys, 2011, 109: 084108

21 Jiang W, Cai W, Lin Z, et al. Effects of Nd-doping on optical and photovoltaic properties of barium titanate thin films prepared by sol-gel method. Mater Res Bull, 2013, 48: 3092-3097

22 Cao D, Wang C, Zheng F, et al. High-efficiency ferroelectric-film solar cells with an n-type $\mathrm{Cu}_{2} \mathrm{O}$ cathode buffer layer. Nano Lett, 2012, 12: 2803-2809

23 Cao D, Wang C, Zheng F, et al. Understanding the nature of remnant polarization enhancement, coercive voltage offset and timedependent photocurrent in ferroelectric films irradiated by ultraviolet light. J Mater Chem, 2012, 22: 12592 
24 Yang X, Su X, Shen M, et al. Enhancement of photocurrent in ferroelectric films via the incorporation of narrow bandgap nanoparticles. Adv Mater, 2012, 24: 1202-1208

25 Cao D, Zhang H, Fang L, et al. Polarization effect on the photocurrent of Pt sandwiched multi-crystalline ferroelectric films. Mater Chem Phys, 2011, 129: 783-786

26 Park J, Won Ahn C, Won Kim I. Photocurrent of lead-free $\left(\mathrm{K}_{0.5} \mathrm{Na}_{0.5}\right)\left(\mathrm{Mn}_{0.005} \mathrm{Nb}_{0.995}\right) \mathrm{O}_{3}$ ferroelectric nanotubes. J Appl Phys, 2012, 112: 014312

27 Park J, Won SS, Ahn CW, et al. Ferroelectric photocurrent effect in polycrystalline lead-free $\left(\mathrm{K}_{0.5} \mathrm{Na}_{0.5}\right)\left(\mathrm{Mn}_{0.005} \mathrm{Nb}_{0.995}\right) \mathrm{O}_{3}$ thin film. J Am Ceram Soc, 2013, 96: 146-150

28 Guo R, You L, Chen L, et al. Photovoltaic property of $\mathrm{BiFeO}_{3}$ thin films with $109^{\circ}$ domains. Appl Phys Lett, 2011, 99: 122902

29 Yan F, Chen G, Lu L, et al. Dynamics of photogenerated surface charge on $\mathrm{BiFeO}_{3}$ films. ACS Nano, 2012, 6: 2353-2360

30 Nechache R, Harnagea C, Licoccia S, et al. Photovoltaic properties of $\mathrm{Bi}_{2} \mathrm{FeCrO}_{6}$ epitaxial thin films. Appl Phys Lett, 2011, 98: 202902

31 Nechache R, Huang W, Li S, et al. Photovoltaic properties of $\mathrm{Bi}_{2} \mathrm{FeCrO}_{6}$ films epitaxially grown on (100)-oriented silicon substrates. Nanoscale, 2016, 8: 3237-3243

32 Ji W, Yao K, Liang YC. Evidence of bulk photovoltaic effect and large tensor coefficient in ferroelectric $\mathrm{BiFeO}_{3}$ thin films. Phys Rev B, 2011, 84: 094115

33 Meng W, Saparov B, Hong F, et al. Alloying and defect control within chalcogenide perovskites for optimized photovoltaic application. Chem Mater, 2016, 28: 821-829

34 Zhang W, Yang MM, Liang $\mathrm{X}$, et al. Piezostrain-enhanced photovoltaic effects in $\mathrm{BiFeO}_{3} / \mathrm{La}_{0.7} \mathrm{Sr}_{0.3} \mathrm{MnO}_{3} / \mathrm{PMN}-\mathrm{PT}$ heterostructures. Nano Energy, 2015, 18: 315-324

35 Bousquet E, Dawber M, Stucki N, et al. Improper ferroelectricity in perovskite oxide artificial superlattices. Nature, 2008, 452: 732-736

36 Senn MS, Bombardi A, Murray CA, et al. Negative thermal expansion in hybrid improper ferroelectric Ruddlesden-Popper perovskites by symmetry trapping. Phys Rev Lett, 2015, 114: 035701

37 Choi T, Lee S, Choi YJ, et al. Switchable ferroelectric diode and photovoltaic effect in $\mathrm{BiFeO}_{3}$. Science, 2009, 324: 63-66

38 Young SM, Zheng F, Rappe AM. First-principles calculation of the bulk photovoltaic effect in bismuth ferrite. Phys Rev Lett, 2012, 109: 236601

39 Chen B, Zuo Z, Liu Y, et al. Tunable photovoltaic effects in transparent $\mathrm{Pb}\left(\mathrm{Zr}_{0.53}, \mathrm{Ti}_{0.47}\right) \mathrm{O}_{3}$ capacitors. Appl Phys Lett, 2012, 100: 173903

40 Daranciang D, Highland MJ, Wen H, et al. Ultrafast photovoltaic response in ferroelectric nanolayers. Phys Rev Lett, 2012, 108: 087601

41 Gou GY, Bennett JW, Takenaka H, et al. Post density functional theoretical studies of highly polar semiconductive $\mathrm{Pb}\left(\mathrm{Ti}_{1-x} \mathrm{Ni}_{x}\right) \mathrm{O}_{3-x}$ solid solutions: effects of cation arrangement on band gap. Phys Rev B, 2011, 83: 205115

42 Berger RF, Neaton JB. Computational design of low-band-gap double perovskites. Phys Rev B, 2012, 86: 165211

43 Zheng $\mathrm{T}$, Deng $\mathrm{H}$, Zhou W, et al. Bandgap modulation and magnetic switching in $\mathrm{PbTiO}_{3}$ ferroelectrics by transition elements doping. Ceramics Int, 2016, 42: 6033-6038

44 Choi WS, Chisholm MF, Singh DJ, et al. Wide bandgap tunability in complex transition metal oxides by site-specific substitution. Nat Commun, 2012, 3: 689

45 Wang F, Grinberg I, Rappe AM. Semiconducting ferroelectric photovoltaics through $\mathrm{Zn}^{2+}$ doping into $\mathrm{KNbO}_{3}$ and polarization rotation. Phys Rev B, 2014, 89: 235105 light-absorbing ferroelectric and photovoltaic materials. Nature, 2013, 503: 509-512

47 Nechache R, Harnagea C, Li S, et al. Bandgap tuning of multiferroic oxide solar cells. Nat Photon, 2014, 9: 61-67

48 Zhang YG, Zheng HW, Zhang JX, et al. Photovoltaic effects in $\mathrm{Bi}_{4} \mathrm{Ti}_{3} \mathrm{O}_{12}$ thin film prepared by a sol-gel method. Mater Lett, 2014, 125: 25-27

49 Tu CS, Chen CS, Chen PY, et al. Enhanced photovoltaic effects in A-site samarium doped $\mathrm{BiFeO}_{3}$ ceramics: the roles of domain structure and electronic state. J Eur Ceramic Soc, 2016, 36: 1149-1157

50 Puli VS, Pradhan DK, Katiyar RK, et al. Photovoltaic effect in transition metal modified polycrystalline $\mathrm{BiFeO}_{3}$ thin films. J Phys D-Appl Phys, 2014, 47: 075502

51 Wang H, Gou G, Li J. Ruddlesden-Popper perovskite sulfides $\mathrm{A}_{3} \mathrm{~B}_{2} \mathrm{~S}_{7}$ : a new family of ferroelectric photovoltaic materials for the visible spectrum. Nano Energy, 2016, 22: 507-513

52 Saeki M, Yajima Y, Onoda M. Preparation and crystal structures of new barium zirconium sulfides, $\mathrm{Ba}_{2} \mathrm{ZrS}_{4}$ and $\mathrm{Ba}_{3} \mathrm{Zr}_{2} \mathrm{~S}_{7}$. J Solid State Chem, 1991, 92: 286-294

53 Shvydka D, Karpov VG. Nanodipole photovoltaics. Appl Phys Lett, 2008, 92: 053507

54 Jha R, Liu X, Wieland K, et al. Capacitance-voltage characterization of solar cells with CdS in CdTe matrix. MRS Proc, 2010, 1260: 1260-T13-04

55 Huang F, Liu X. A ferroelectric-semiconductor-coupled solar cell with tunable photovoltage. Appl Phys Lett, 2013, 102: 103501

56 Huang F, Liu X, Wang W. A CdS nanodipole solar cell. Prog Photovolt-Res Appl, 2015, 23: 319-330

57 Schmidt ME, Blanton SA, Hines MA, et al. Polar CdSe nanocrystals: Implications for electronic structure. J Chem Phys, 1997, 106: 5254-5259

58 Blanton SA, Leheny RL, Hines MA, et al. Dielectric dispersion measurements of CdSe nanocrystal colloids: observation of a permanent dipole moment. Phys Rev Lett, 1997, 79: 865-868

59 Shim M, Guyot-Sionnest P. Permanent dipole moment and charges in colloidal semiconductor quantum dots. J Chem Phys, 1999, 111: 6955-6964

60 Hao LZ, Gao W, Liu YJ, et al. High-performance n- $\mathrm{MoS}_{2} / \mathrm{i}-\mathrm{SiO}_{2}$ /p-Si heterojunction solar cells. Nanoscale, 2015, 7: 8304-8308

61 Fan Z, Xiao J, Yao K, et al. Ferroelectric polarization relaxation in $\mathrm{Au} / \mathrm{Cu}_{2} \mathrm{O} / \mathrm{ZnO} / \mathrm{BiFeO}_{3} / \mathrm{Pt}$ heterostructure. Appl Phys Lett, 2015, 106: 102902

62 Dong W, Guo Y, Guo B, et al. Photovoltaic properties of $\mathrm{BiFeO}_{3}$ thin film capacitors by using Al-doped zinc oxide as top electrode. Mater Lett, 2013, 91: 359-361

63 Zheng F, Xin Y, Huang W, et al. Above 1\% efficiency of a ferroelectric solar cell based on the $\mathrm{Pb}(\mathrm{Zr}, \mathrm{Ti}) \mathrm{O}_{3}$ film. J Mater Chem A, 2014, 2: $1363-1368$

64 Zheng F, Zhang P, Wang X, et al. Photovoltaic enhancement due to surface-plasmon assisted visible-light absorption at the inartificial surface of lead zirconate-titanate film. Nanoscale, 2014, 6 : 2915-2921

65 Nalwa KS, Carr JA, Mahadevapuram RC, et al. Enhanced charge separation in organic photovoltaic films doped with ferroelectric dipoles. Energy Environ Sci, 2012, 5: 7042

66 Garbugli M, Porro M, Roiati V, et al. Light energy harvesting with nano-dipoles. Nanoscale, 2012, 4: 1728-1733

67 Fan Z, Yao K, Wang J. Photovoltaic effect in an indium-tin-oxide/ $\mathrm{ZnO} / \mathrm{BiFeO}_{3} / \mathrm{Pt}$ heterostructure. Appl Phys Lett, 2014, 105: 162903

68 Pan DF, Bi GF, Chen GY, et al. Polarization-dependent interfacial 
coupling modulation of ferroelectric photovoltaic effect in PZTZnO heterostructures. Sci Rep, 2016, 6: 22948

69 Chatterjee S, Bera A, Pal AJ. p-i-n Heterojunctions with $\mathrm{BiFeO}_{3}$ perovskite nanoparticles and p- and n-type oxides: photovoltaic properties. ACS Appl Mater Interfaces, 2014, 6: 20479-20486

70 Zhang JX, Zheng HW, Zhang YG, et al. Photovoltaic effect of a bilayer film with $\mathrm{Bi}_{4} \mathrm{Ti}_{3} \mathrm{O}_{12} / \mathrm{BiFeO}_{3}$ heterostructure. Mater Lett, 2015, 156: $98-100$

71 Nie C, Zhao S, Bai Y, et al. The ferroelectric photovoltaic effect of $\mathrm{BiCrO}_{3} / \mathrm{BiFeO}_{3}$ bilayer composite films. Ceramics Int, 2016, 42: 14036-14040

72 Sharma S, Tomar M, Kumar A, et al. Photovoltaic effect in $\mathrm{BiFeO}_{3} / \mathrm{BaTiO}_{3}$ multilayer structure fabricated by chemical solution deposition technique. J Phys Chem Solids, 2016, 93: 63-67

73 Chakrabartty J, Nechache R, Harnagea C, et al. Enhanced photovoltaic properties in bilayer $\mathrm{BiFeO}_{3} / \mathrm{Bi}-\mathrm{Mn}-\mathrm{O}$ thin films. Nanotechnology, 2016, 27: 215402

74 Wu F, Guo Y, Guo B, et al. Photovoltaic effect of a bilayer thin film with $\left(\mathrm{Na}_{0.5} \mathrm{Bi}_{0.5}\right)_{1-x} \mathrm{Ba}_{x} \mathrm{TiO}_{3} / \mathrm{BiFeO}_{3}$ heterostructure. J Phys D-Appl Phys, 2013, 46: 365304

75 Dong W, Guo Y, Guo B, et al. Enhanced photovoltaic effect in $\mathrm{BiVO}_{4}$ semiconductor by incorporation with an ultrathin $\mathrm{BiFeO}_{3}$ ferroelectric layer. ACS Appl Mater Interfaces, 2013, 5: 6925-6929

76 Chakrabartty J, Nechache R, Li S, et al. Photovoltaic properties of multiferroic $\mathrm{BiFeO}_{3} / \mathrm{BiCrO}_{3}$ heterostructures. J Am Ceram Soc, 2014, 97: 1837-1840

77 Chakrabartty JP, Nechache R, Harnagea C, et al. Photovoltaic effect in multiphase Bi-Mn-O thin films. Opt Express, 2014, 22: A80-89

78 Zhou Y, Fang L, You L, et al. Photovoltaic property of domain engineered epitaxial $\mathrm{BiFeO}_{3}$ films. Appl Phys Lett, 2014, 105: 252903

79 Kojima A, Teshima K, Shirai Y, et al. Organometal halide perovskites as visible-light sensitizers for photovoltaic cells. J Am Chem Soc, 2009, 131: 6050-6051

80 Stranks SD, Eperon GE, Grancini G, et al. Electron-hole diffusion lengths exceeding 1 micrometer in an organometal trihalide perovskite absorber. Science, 2013, 342: 341-344

81 Liu H, Chen J, Ren Y, et al. Large photovoltage and controllable photovoltaic effect in $\mathrm{PbTiO}_{3}-\mathrm{Bi}\left(\mathrm{N}_{\mathrm{i} 2 / 3+x} \mathrm{Nb}_{1 / 3-x}\right) \mathrm{O}_{3-\delta}$ Ferroelectrics. Adv Electron Mater, 20151: 1400051

82 Inoue R, Ishikawa S, Imura R, et al. Giant photovoltaic effect of ferroelectric domain walls in perovskite single crystals. Sci Rep, 2015, 5: 14741

83 Hu Y, Wang C, Tang Y, et al. Three-dimensional self-branching anatase $\mathrm{TiO}_{2}$ nanorods with the improved carrier collection for $\mathrm{Sr}-$ $\mathrm{TiO}_{3}$-based perovskite solar cells. J Alloys Compounds, 2016, 679: 32-38

84 Frost JM, Butler KT, Brivio F, et al. Atomistic origins of high-performance in hybrid halide perovskite solar cells. Nano Lett, 2014, 14: $2584-2590$

85 Fan Z, Xiao J, Sun $\mathrm{K}$, et al. Ferroelectricity of $\mathrm{CH}_{3} \mathrm{NH}_{3} \mathrm{PbI}_{3}$ perovskite. J Phys Chem Lett, 2015, 6: 1155-1161
86 Filippetti A, Delugas P, Saba MI, et al. Entropy-suppressed ferroelectricity in hybrid lead-iodide perovskites. J Phys Chem Lett, 2015, 6: 4909-4915

87 Stoumpos CC, Malliakas CD, Kanatzidis MG. Semiconducting tin and lead iodide perovskites with organic cations: phase transitions, high mobilities, and near-infrared photoluminescent properties. Inorg Chem, 2013, 52: 9019-9038

88 Kutes Y, Ye L, Zhou Y, et al. Direct observation of ferroelectric domains in solution-processed $\mathrm{CH}_{3} \mathrm{NH}_{3} \mathrm{PbI}_{3}$ perovskite thin films. J Phys Chem Lett, 2014, 5: 3335-3339

89 Wei J, Zhao Y, Li H, et al. Hysteresis analysis based on the ferroelectric effect in hybrid perovskite solar cells. J Phys Chem Lett, 2014, 5: $3937-3945$

90 Wang F, Meng D, Li X, et al. Influence of annealing temperature on the crystallization and ferroelectricity of perovskite $\mathrm{CH}_{3} \mathrm{NH}_{3} \mathrm{PbI}_{3}$ film. Appl Surface Sci, 2015, 357: 391-396

91 Zhao P, Xu J, Ma C, et al. Spontaneous polarization behaviors in hybrid halide perovskite film. Scripta Mater, 2015, 102: 51-54

92 Xiao Z, Yuan Y, Shao Y, et al. Giant switchable photovoltaic effect in organometal trihalide perovskite devices. Nat Mater, 2014, 14: 193-198

93 Beilsten-Edmands J, Eperon GE, Johnson RD, et al. Non-ferroelectric nature of the conductance hysteresis in $\mathrm{CH}_{3} \mathrm{NH}_{3} \mathrm{PbI}_{3}$ perovskite-based photovoltaic devices. Appl Phys Lett, 2015, 106: 173502

94 Kim GY, Oh SH, Nguyen BP, et al. Efficient carrier separation and intriguing switching of bound charges in inorganic-organic lead halide solar cells. J Phys Chem Lett, 2015, 6: 2355-2362

95 Leguy AMA, Frost JM, McMahon AP, et al. The dynamics of methylammonium ions in hybrid organic-inorganic perovskite solar cells. Nat Commun, 2015, 6: 7124

96 Sherkar TS, Jan Anton Koster L. Can ferroelectric polarization explain the high performance of hybrid halide perovskite solar cells? Phys Chem Chem Phys, 2016, 18: 331-338

97 Liu S, Zheng F, Koocher NZ, et al. Ferroelectric domain wall induced band gap reduction and charge separation in organometal halide perovskites. J Phys Chem Lett, 2015, 6: 693-699

98 Yamada Y, Nakamura T, Yasui S, et al. Measurement of transient photoabsorption and photocurrent of $\mathrm{BiFeO}_{3}$ thin films: evidence for long-lived trapped photocarriers. Phys Rev B, 2014, 89: 035133

Acknowledgments This work was supported by the National Basic Research Program of China (2013CB921904 and 2016YFB0401003), and the National Natural Science Foundation of China (11574009 and 61575005).

Author contributions Liu Y wrote this manuscript under the guidance of Wang S; Xiao L and Chen Z contributed to the general discussion and article revision.

Conflict of interest The authors declare that they have no conflict of interest. 

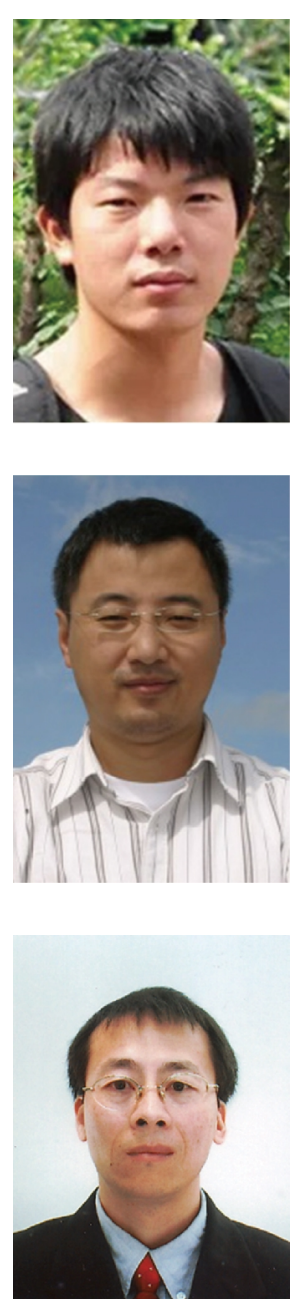

Yang Liu is a PhD candidate at the Department of Physics, Peking University, China. He joined Prof. Shufeng Wang's Ultrafast Spectroscopy group in 2014. Now his main project is on the photophysics of perovskite photovoltaic materials.
Shufeng Wang got his PhD degree in 2001, from the Department of Physics, Peking University, China, under the guidance of Prof. Qihuang Gong. He worked as a postdoctoral researcher in the group of Prof. Dana D. Dlott at the University of Illinois at Urbana-Champaign (USA) between 2001 and 2004. Now he is an associate professor at the Department of Physics, Peking University. His research interests are on nonlinear optics and ultrafast dynamics of photovoltaic materials and devices.

Lixin Xiao is a professor of the Department of Physics, Peking University (China) since 2011. He received his PhD in applied chemistry from the University of Tokyo (Japan) in 2000. He has been working on organic optoelectronics materials and devices, especially on photovoltaic (PV) and organic light-emitting devices (OLED).

\section{铁电光伏的应用}

刘洋 ${ }^{1}$, 王树峰 ${ }^{1,2^{*}}$, 陈志坚 ${ }^{1,3}$, 肖立新 ${ }^{1,3^{*}}$

摘要 具有反常光伏效应的铁电材料是光伏研究的重点之一. 本文综述了铁电材料在光伏应用中的研究进展, 包括铁电性的物理基础、铁 电薄膜的性质、铁电光伏效应的特点和内在机制、铁电材料太阳电池等. 这些发现对于进一步提高光伏电池的效率具有重要意义. 